\title{
Prescription Errors with Chemotherapy: Quality Improvement through Standardized Order Templates
}

\author{
Aline Saad ${ }^{1 *}$, Caroline A. Der-Nigoghossian ${ }^{2}$, Rachel Njeim ${ }^{2}$, Riwa Sakr ${ }^{3}$, Pascale \\ Salameh $^{4}$, Marcel Massoud ${ }^{3}$
}

\begin{abstract}
Background: Despite the existence of established guidelines advocating the use and value of chemotherapy order templates, chemotherapy orders are still handwritten in many hospitals in Lebanon. This manuscript describes the implementation of standardized chemotherapy order templates (COT) in a Lebanese tertiary teaching hospital through multiple steps. Initial Assessment: An initial assessment was conducted through a retrospective appraisal of completeness of handwritten chemotherapy orders for 100 adult patients to serve as a baseline for the project and identify parameters that might afford improvement. Choice of solution: Development of over 300 standardized pre-printed COTs based on the National Comprehensive Cancer Network templates and adapted to the practice culture and patient population. Implementation: The COTs were implemented, using Kotter's 8-step model for leading change, by engaging health care providers, and identifying and removing barriers. Evaluation: Assessment of physicians' compliance with the new practice (122 orders assessed) was completed through two phases and allowed for the identification of areas of improvement. Lessons Learned: Overall, COT implementation showed an average improvement in order completion from $49.5 \%$ (handwritten orders) to $77.6 \%$ (phase 1-COT) to $87.6 \%$ (phase 2-COT) reflecting an increase of $38.1 \%$ between baseline and phase 2 and demonstrating that chemotherapy orders completeness was improved by pre-printed COT. As many of the hospitals in Lebanon are moving towards standardized COTs and computerized physician order entry (CPOE) in the next few years, this study provides a prototype for the successful implementation of COT and demonstrates their role in promoting quality improvement of cancer care.
\end{abstract}

Keywords: Patient and medication safety - quality improvement - standardization of care - and chemotherapy order

Asian Pac J Cancer Prev, 17 (4), 2329-2336

\section{Introduction}

Safety concerns related to medication errors involving antineoplastic agents are a major focus of quality improvement initiatives in health care settings (Shaikh et al., 2004). Administration errors of antineoplastics can be fatal due to these agents' inherent toxicity and narrow therapeutic indices (Dumasia et al., 2006). In addition, chemotherapy is frequently administered to patients who are physiologically compromised by their underlying disease, leading to higher risk of complications (Harris and Northfelt, 2005).

It is estimated that 500 deaths occur annually in the United States (U.S.) due to chemotherapy-related medication errors making antineoplastic agents the second most common cause of fatal medication errors (Phillips et al., 2001; Northfelt et al., 2003). A review of the medical records of 1262 adult and 117 pediatric cancer patients showed that $7 \%$ and $19 \%$ of adult and pediatric outpatient clinic visits, respectively, were associated with medication errors most commonly occurring during the administration of chemotherapy agents. Of those involving adults, 55 medication errors had the potential to harm the patient and 11 caused harm (Walsh et al., 2009). Similarly, a study in Spain reported medication errors among $17.2 \%$ of patients receiving chemotherapy. These errors were identified in the prescription $(75.7 \%)$, preparation $(21 \%)$, dispensing $(1.8 \%)$, administration $(1.1 \%)$ and follow-up phases $(0.4 \%)$ (Serrano-fabia et al., 2010). In France, a study reported an incidence of $5.2 \%$ of chemotherapy medication errors, $48 \%$ of which were attributed to incomplete prescriptions (Ranchon et al., 2011). In Sweden, a study aiming to identify the characteristics of medication errors involving chemotherapeutic agents stated that $42 \%$ of these errors occurred in the prescribing phase, another $42 \%$ occurred in the preparation phase and $16 \%$ in the administration phase (Fyhr and Akselsson, 2012). In the U.S., Dumasia et al. has shown that handwritten chemotherapy orders lacked some crucial prescribing information such as chemotherapy dose, number of doses and route of administration, with a

${ }^{1}$ Department of Pharmacy Practice School of Pharmacy Lebanese American University, ${ }^{2}$ Lebanese American University Byblos, ${ }^{3}$ Holy Spirit University of Kaslik Jounieh, ${ }^{4}$ Department of Pharmacy Practice School of Pharmacy Lebanese American University Byblos, Lebanon*For correspondence: aline.saad@lau.edu.lb 
frequent use of abbreviations and trailing zeros (Dumasia et al., 2006).

In Lebanon, documented information on chemotherapyrelated errors is limited. One study of health care providers' perception of drug administration safety in pediatric oncology patients in Lebanon showed that reports of medication errors are wide-ranging (between $1.5 \%$ and $90 \%$ ) depending on the institutions where data were collected and the methods used for error identification. Results from the same study also showed that the risk of medication errors with potential harm is three times higher for children as compared to adults, especially those receiving chemotherapy (Harris et al., 2014).

Remedial actions to minimize chemotherapy errors have been proposed and this goal became an evolving process in the practice of oncology. For instance, health care institutions have thoroughly reviewed and subsequently revised their chemotherapy policies and procedures in order to improve safety and elevate the standards of care (Birner et al., 2006). Changes have included developing computerized pre-printed orders, using Computerized Physician Order Entry (CPOE) that is specifically designed for chemotherapy, and implementing Clinical Decision Support Systems (CDSS). Other methods have included physicians, nurses and pharmacists double-checking chemotherapy orders, continuing education and staff training, providing instant access to medication information, improving systems of reporting errors, and standardizing medication preparation, labeling and dispensing (Schulmeister, 2006). In addition, literature data have consistently confirmed that standardized chemotherapy order forms have enhanced the prescribing patterns, prescription completeness, and decreased the potential for medication errors (Dumasia et al., 2006).

The National Comprehensive Cancer Network (NCCN) Chemotherapy Order Templates (COT) was launched in 2008 to complement the NCCN Clinical Practice Guidelines in Oncology. They delineate antineoplastic agents and associated supportive care agents, monitoring and safety parameters, and instructions for self-administered agents. Through COT, NCCN sought to enhance patient safety by reducing medication errors, anticipating and managing adverse events, and standardizing care (Li et al., 2013). The American Society of Clinical Oncology (ASCO) and Oncology Nursing Society (ONS) have also released a set of 31 standards in 2009 for the safe administration of chemotherapy in the adult outpatient setting. These standards are intended to improve patient safety, reduce the risk of medication errors, increase clinical efficiency, and provide a framework for best practices. The ASCO/ONS recommendations call for "standardized approaches" and recommend that all chemotherapy orders be written using standardized, preprinted practice forms (Dreyfuss, 2010). In addition, the American Society of Health-System Pharmacists (ASHP) and the Institute for Safe Medication Practices (ISMP) have published guidelines for Chemotherapy Order Forms, and set recommendations for pre-printed chemotherapy orders (ASHP, 2002; ISMP 2014). ASHP also proposed to "mandate the use of pre-printed order forms that standardize practice and force functions" as the first strategy to prevent cancer chemotherapy errors (Kloth, 2010). ISMP advocated standard order sets to integrate and coordinate evidence based care by communicating best practices through multiple disciplines and reduce the potential for medication errors using integrated safety alerts and reminders (ISMP, 2014).

Despite these established guidelines, the majority of hospitals in Lebanon continue to have their chemotherapy orders handwritten by the oncologists. Although COT have been the standard of care for years in the U.S., existing barriers have delayed their adoption in Lebanon. Thus, the objective of this study was to demonstrate a step-bystep approach to a successful implementation of COT in a tertiary teaching hospital in Lebanon through the improved completeness of chemotherapy orders.

\section{Initial Assessment/Choice of Solution/ Implementation}

\section{Setting}

The study was conducted in a 230-bed teaching hospital in Lebanon. Six attending medical oncologists, three hematology-oncology fellows, three rotating internal medicine residents and four medical interns oversee the care of patients in a 21-bed inpatient adult unit and a 20bed outpatient adult unit for hematology/oncology. Until April 2011, chemotherapy orders were handwritten by oncologists. Introducing pre-printed COT was therefore considered which included pertinent patient information, chemotherapy and supportive care data, with the main goal of decreasing medication errors through completeness of chemotherapy orders.

\section{Procedure}

A multi-phase project that spanned over 4 years was initiated, and included: $i$ ). Step 1: Retrospective review of handwritten chemotherapy orders, through a predesigned data collection sheet, for 100 patients served as baseline for the assessment of the way orders were written (completed in 2010); ii). Step 2: Development of standardized preprinted COT based on the NCCN templates and the ASCO/ ONS standards and adapted to the practice culture and patient population (completed in 2010-2011); iii). Step 3: Implementation of the standardized COT requiring various educational sessions (completed in 2011); iv). Step 4: Postimplementation phase with a retrospective review of preprinted COT: data collection form used in step 1 was used to assess physicians' compliance with the new practice and identify areas of improvement. This activity was completed directly after implementation (phase 1- 20112012) for 51 randomly selected chemotherapy orders, and 2 years after for 71 randomly selected orders (phase 2-2013-2014); v). Step 5: Survey of hospitals in Lebanon for their use of handwritten or pre-printed chemotherapy orders in lieu of the scarcity of published literature around this topic in the country (completed in 2014).

Meetings with oncology physicians and nurses were scheduled before and during the implementation of the standardized COT to reinforce their engagement and address any foreseeable challenges. The study was approved by the hospital's Institutional Review Board. 


\section{Data collection sheet}

The data collection sheet used during Steps 1 and 4 (baseline, phases 1 and 2) is the same and captured information pertaining to patients' demographics, chemotherapy protocol details, chemotherapy medication parameters, and supportive care. Demographics included patient's identification number; date of birth; height and weight for body surface area calculation; allergies; serum creatinine ( $\mathrm{SCr}$ ); diagnosis; and stage of the cancer. Chemotherapy protocols captured the protocol name and primary reference; cycle number; total number of cycles; and chemotherapy start date. Chemotherapy medication parameters included the drug name used to list chemotherapy (generic versus brand); dosage regimen; route; method (continuous infusion, intravenous push...); frequency and duration of administration; diluent type and volume; and sequence of drug administration, when applicable. Supportive care data encompassed antiemetics agents prescribed, including 5-HT3 receptor antagonists; steroids and other; pre-medications; and hydration requirements. Chemotherapy regimens and antiemetics use were assessed for congruence with the NCCN recommendations based on diagnosis/staging of the patient and emetogenic classification of the chemotherapy used, respectively.

Step 5 consisted of a survey questionnaire that was sent to 30 hospitals in Lebanon selected from 163 hospitals contracted with the Ministry of Public Health. The hospitals were chosen to represent the two available sectors (private and public), geographical areas (the six Lebanese districts), and varying hospital bed capacities. The survey was completed by the hospital pharmacists and gathered information including basic demographic data regarding the hospital, whether chemotherapy orders are handwritten or pre-printed, the year the hospital started implementing pre-printed COT and any future plans regarding this matter (including pre-printed COT or CPOE).

\section{Statistical analysis}

Descriptive analysis was performed to estimate the frequency of orders completeness. Variables were summarized using frequencies and percentages. The association between categorical variables was evaluated using Pearson $\chi^{2}$ test or Fisher's exact test where the expected cell count was less than 5 . A priori p-value of 0.05 or less was considered to be statistically significant. Moreover, a trend analysis was performed to assess the linear increase or decrease in percentages, and p-values were reported.

\section{Evaluation}

\section{Step 1- initial assessment}

During the baseline phase, chemotherapy orders for 100 patients were evaluated through the predefined criteria of the data collection sheets. Before implementation of standardized COT, handwritten orders lacked patient's allergy information in $98 \%$ of cases, and protocol specific information such as protocol name, reference, total number of cycles, and start date of chemotherapy in $95 \%, 100 \%, 94 \%$, and $96 \%$ of reviewed orders, respectively. Chemotherapy agents were mostly provided in brand names (62\%) with no dosing in $\mathrm{mg} / \mathrm{m}^{2}$ or $\mathrm{mg} / \mathrm{kg}$ specified. The chemotherapy agent administration route, method, and frequency were documented in $73 \%, 55 \%$ and $52 \%$ of reviewed orders, respectively. The duration of infusion of chemotherapy agents was present in $35 \%$ of cases. When multiple chemotherapy agents were administered in a regimen, no specified sequence of administration was provided. Regimens conformed to the NCCN recommendations for the treatment of cancers in $71 \%$ of cases. Anti-emetics were not detailed according to administration time in relation to chemotherapy. All patients received a combination of a steroid and 5HT3 antagonist in addition to other anti-emetic agents such as metoclopramide and promethazine. Prescribed anti-emetic regimens were in compliance with the NCCN guidelines' recommendations in only $35 \%$ of reviewed orders (Tables 1,2 , and 3).

\section{Steps 2 and 3-Choice of Solution and Implementation:}

The baseline status findings were critical and constituted a driving force in creating a sense of urgency to implement standardized pre-printed COT. Accordingly, a leading group of an attending oncologist, medical residents, pharmacist and pharmacy student joined efforts in developing the pre-printed COT. A total of 307 orders were developed over a period of six months, with 85 orders covering the hematologic diseases and 222 orders covering the oncologic diseases based on the most commonly treated types of cancer diagnoses at the hospital. These orders were in line with the NCCN proposed template and the ASCO/ONS standards but were also modified to fit the Lebanese practice and patient population. The developed COT prompted the prescribers to complete patient, chemotherapy, and supportive care variables necessary for chemotherapy order completeness. To accommodate the practice setting, $\mathrm{SCr}$ was not included in the COT as a patient's $\mathrm{SCr}$ is ordered directly prior to chemotherapy infusion and the patient's receipt of chemotherapy would be contingent on the $\mathrm{SCr}$ results and renal function. As such, $\mathrm{SCr}$ would not be featured on the order that was pre-filled by the prescriber. Orders were kept simple and easy to handle in order to address the language barrier with some of the nurses who were French educated. Orders were reviewed by different committees including the hospital Pharmacy \& Therapeutics committee in order to ensure accuracy of information and gain acceptance and ownership by various stakeholders.

\section{Step 4-Post-implementation assessment-Phase 1:}

Within six months of implementation, 51 chemotherapy orders were evaluated for completeness. Compared to the baseline status, there was a significant increase in order completeness with: improved allergies documentation (from $2 \%$ to $46 \%, \mathrm{p}<0.001$ ), total number of cycles (from $6 \%$ to $18 \%, \mathrm{p}=0.024)$ and start date of chemotherapy (from $4 \%$ to $71 \%, \mathrm{p}<0.001$ ). Orders statistically regressed on completion of information pertaining to height (from $100 \%$ to $81 \%, \mathrm{p}<0.001$ ), weight (from $100 \%$ to $83 \%$, $\mathrm{p}<0.001$ ), serum creatinine (from $98 \%$ to $2 \%, \mathrm{p}<0.001$ ) 
Aline Saad et al

and cycle number (from $94 \%$ to $57 \%, \mathrm{p}<0.001$ ). As the standardized pre-printed COT systematically provided the protocol name and reference, the chemotherapy agents detailed data such as generic drug name, dose in $\mathrm{mg} / \mathrm{m}^{2}$ or $\mathrm{mg} / \mathrm{kg}$, administration route, method and frequency, infusion duration and diluent type and volume, a notable increase in completeness of orders was achieved between baseline and phase 1 on these parameters that was statistically significant for the evaluated parameters. Improvements could still be implemented to specifying the sequence of chemotherapy agents' administration when applicable $(0 \%$ completed in the baseline status versus $18 \%$ completed in phase $1, \mathrm{p}<0.001)$ and to complying with the NCCN treatment guidelines $(71 \%$ conformed in the baseline status versus $63 \%$ in phase $1, \mathrm{p}=0.303$ ).
For anti-emesis prescribing, a change was not observed in outcomes with the use of standardized orders. In fact, upon comparing with the NCCN recommendations based on emetic potential, the evaluated orders were in line with the guidelines in $4 \%$ of the cases only. Oncologists still use various combinations of steroids, 5-HT3 antagonists and other anti-emetic agents. The noted statistically significant improvement was in pre-medications (from 62\% to $97 \%$, $\mathrm{p}<0.001$ ) and hydration (from $29 \%$ to $87 \%$, p<0.001) as these are now pre-printed in applicable orders (Tables 1 , 2 and 3$)$.

Step 4-Post-implementation assessment- Phase 2:

Within 2 years of implementation and alongside with educational interventions to reinforce compliance

Table 1. Percentage of Order Completeness as Pertaining to Patient Information at Baseline and After Implementation (Phases 1 and 2)

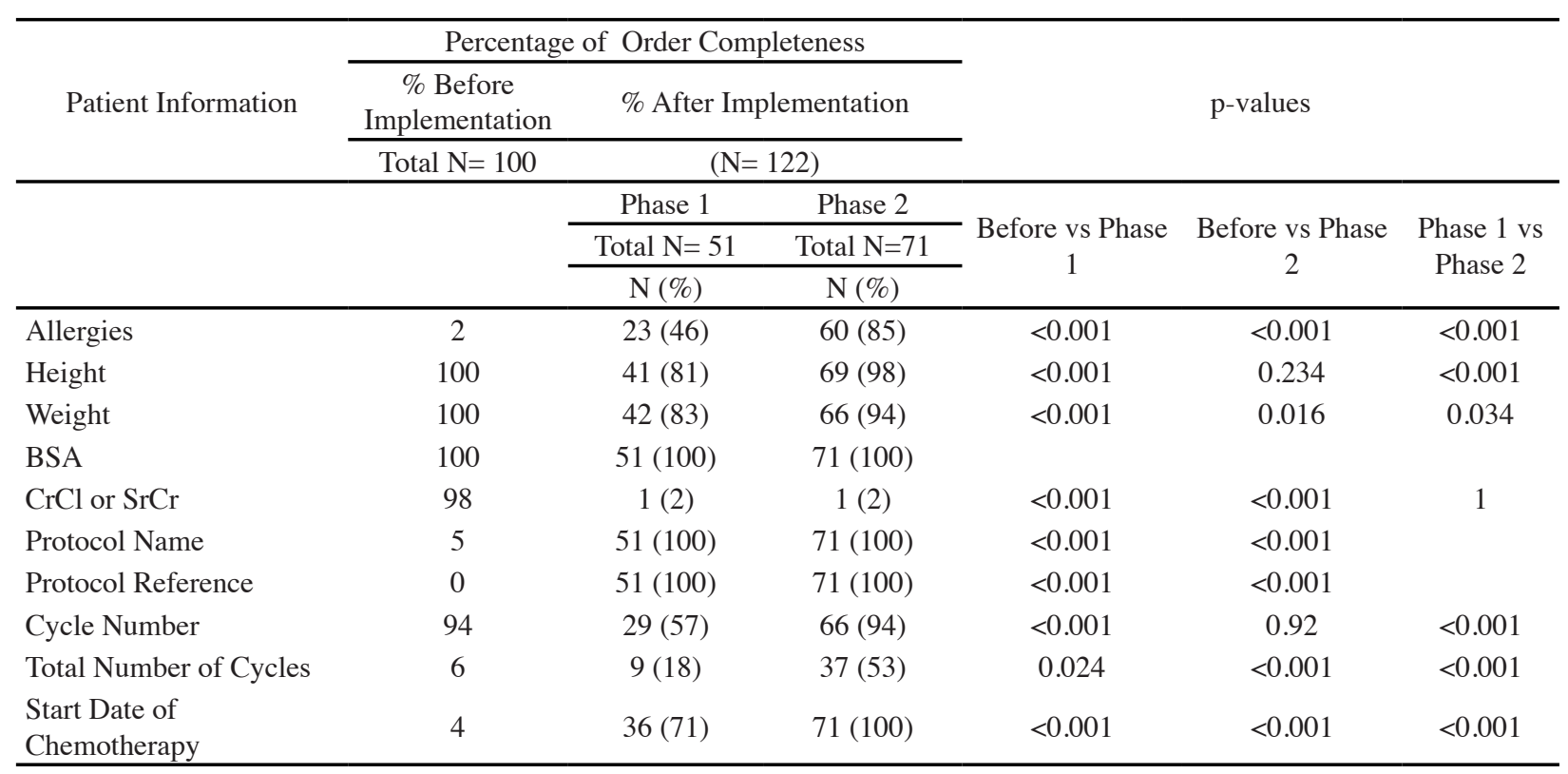

Empty cells reflect the inability to run statistical analysis on the corresponding data

Table 2. Percentage of Order Completeness as Pertaining to Chemotherapy Information at Baseline and After Implementation (Phases 1 and 2)

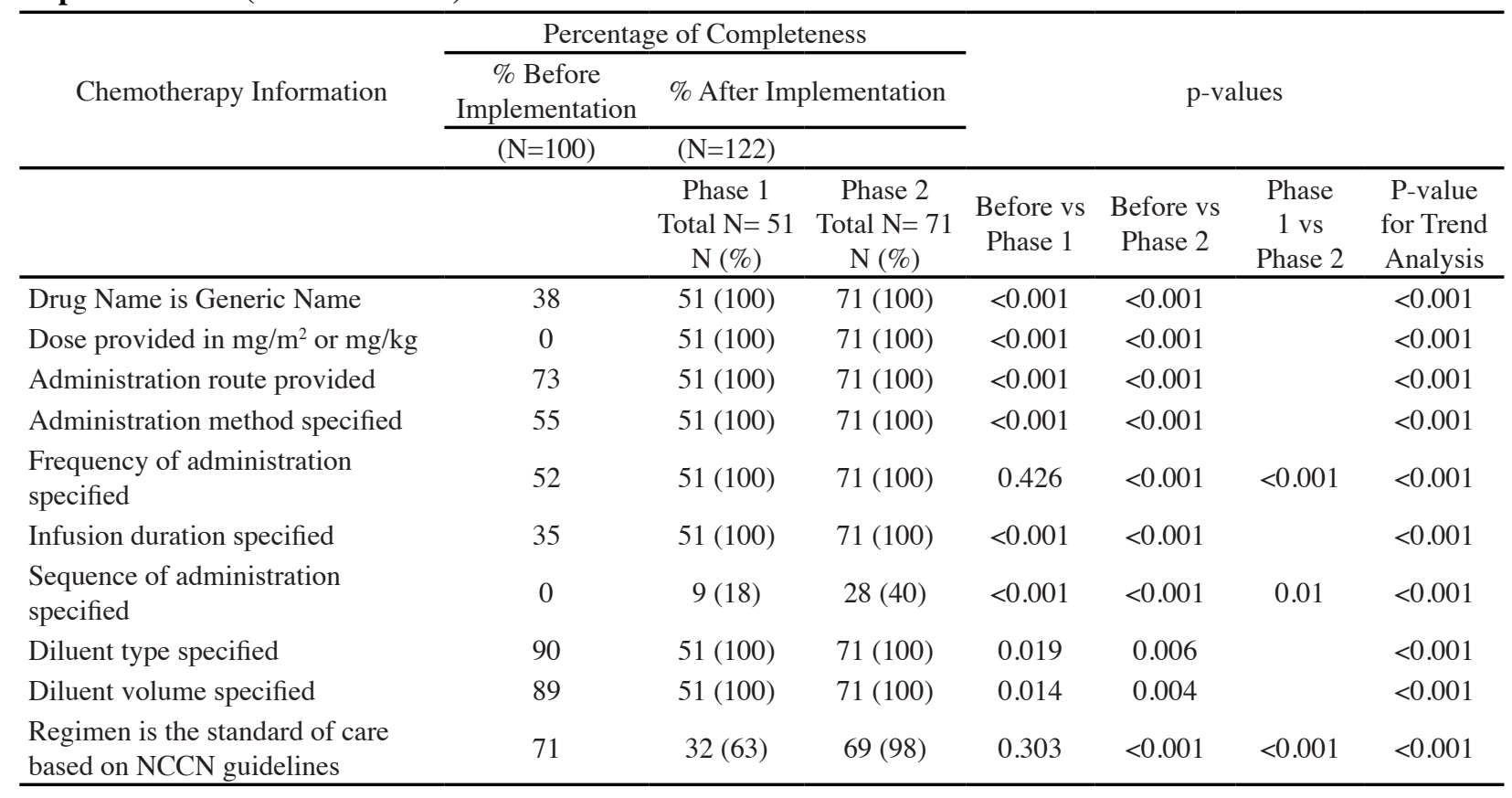


Table 3. Percentage of Order Completeness as Pertaining to Supportive Care Information at Baseline and After Implementation (Phases 1 and 2)

\begin{tabular}{|c|c|c|c|c|c|c|c|}
\hline \multirow{3}{*}{$\begin{array}{l}\text { Supportive Care } \\
\text { Information }\end{array}$} & \multicolumn{3}{|c|}{ Percentage of Completeness } & \multirow{2}{*}{\multicolumn{4}{|c|}{ p-values }} \\
\hline & \multirow{2}{*}{$\begin{array}{c}\% \text { Before } \\
\text { Implementation } \\
(\mathrm{N}=100)\end{array}$} & \multicolumn{2}{|c|}{$\begin{array}{c}\text { \% After Implementation } \\
(\mathrm{N}=122)\end{array}$} & & & & \\
\hline & & $\begin{array}{c}\text { Phase } 1 \\
\text { Total } \mathrm{N}=51 \\
\mathrm{~N}(\%)\end{array}$ & $\begin{array}{c}\text { Phase } 2 \\
\text { Total N=71 } \\
\mathrm{N}(\%) \\
\end{array}$ & $\begin{array}{l}\text { Before vs } \\
\text { Phase } 1\end{array}$ & $\begin{array}{l}\text { Before vs } \\
\text { Phase } 2\end{array}$ & $\begin{array}{c}\text { Phase } 1 \text { vs } \\
\text { Phase } 2\end{array}$ & $\begin{array}{c}\text { P-value } \\
\text { for Trend } \\
\text { Analysis } \\
\end{array}$ \\
\hline $\begin{array}{l}\text { Antiemetics divided } \\
\text { according to }\end{array}$ & 0 & $17(34)$ & $58(82)$ & $<0.001$ & $<0.001$ & $<0.001$ & $<0.001$ \\
\hline \multicolumn{8}{|c|}{ pre-chemo and post-chemo orders } \\
\hline \multicolumn{8}{|c|}{ 5HT3 Antagonist Prescribed: } \\
\hline 1. Granisetron & 27 & $10(20)$ & $30(43)$ & 0.318 & 0.035 & 0.006 & 0.041 \\
\hline 2. Ondansetron & 40 & $35(69)$ & $40(57)$ & 0.001 & 0.035 & 0.169 & 0.028 \\
\hline 3. Tropisetron & 24 & 0 & 0 & $<0.001$ & $<0.001$ & & $<0.001$ \\
\hline 4. None prescribed & 9 & $1(2)$ & $2(3)$ & 0.165 & 0.124 & 1.000 & 0.067 \\
\hline \multicolumn{8}{|l|}{ Steroid Agent Prescribed: } \\
\hline 1. Dexamethasone & 39 & $14(28)$ & $28(40)$ & 0.160 & 0.954 & 0.169 & 0.988 \\
\hline 2. Methylprednisolone & 61 & $28(55)$ & $38(54)$ & 0.471 & 0.329 & 0.88 & 0.317 \\
\hline 3. Prednisone & 0 & 0 & $5(8)$ & 0.0110 .074 & 0.003 & 0.011 & 0.001 \\
\hline 4. None prescribed & 0 & $5(10)$ & 0 & & & & 0.819 \\
\hline \multicolumn{8}{|c|}{ Other Antiemetics Prescribed: } \\
\hline 1. Metoclopramide & 33 & $31(61)$ & $63(89)$ & 0.001 & $<0.001$ & $<0.001$ & $<0.001$ \\
\hline 2. Promethazine & 5 & $8(16)$ & $21(30)$ & 0.034 & $<0.001$ & 0.087 & $<0.001$ \\
\hline 3. Ranitidine & 0 & 0 & $11(16)$ & & $<0.001$ & 0.002 & $<0.001$ \\
\hline 4. None Prescribed & 62 & $12(24)$ & $3(5)$ & $<0.001$ & $<0.001$ & 0.004 & $<0.001$ \\
\hline $\begin{array}{l}\text { Antiemetics confirm } \\
\text { with NCCN guidelines }\end{array}$ & 35 & $2(4)$ & $31(44)$ & $<0.001$ & 0.268 & $<0.001$ & 0.313 \\
\hline $\begin{array}{l}\text { Premedications } \\
\text { specified when needed }\end{array}$ & 62 & $49(97)$ & $66(93)$ & $<0.001$ & $<0.001$ & 0.465 & $<0.001$ \\
\hline $\begin{array}{l}\text { Special hydration } \\
\text { specified when needed }\end{array}$ & 29 & $44(87)$ & $68(95)$ & $<0.001$ & $<0.001$ & 0.124 & $<0.001$ \\
\hline
\end{tabular}

Empty cells reflect the inability to run statistical analysis on the corresponding data

with pre-printed COT, 71 patient orders were reviewed for completeness. Results showed that previously achieved completeness in parameters during phase 1 was maintained, and a statistically significant increase was achieved in the reporting of allergies (from $46 \%$ to $85 \%$, $\mathrm{p}<0.001$ ), cycle number (from $57 \%$ to $94 \%, \mathrm{p}<0.001$ ), total number of cycles (from $18 \%$ to $53 \%, \mathrm{p}<0.001$ ), and start date of chemotherapy (from $71 \%$ to $100 \%$, p < 0.001 ) compared to phase 1 . Previously seen regressions in phase 1 for indicating patients' height, weight, and cycle number were corrected in phase 2 (increase from $81 \%$ to $98 \%, 83 \%$ to $94 \%$, and $57 \%$ to $94 \%$ respectively, all statistically significant). When applicable, the sequence of chemotherapy administration was now specified in $40 \%$ of evaluated cases, and regimens were in congruence with NCCN guidelines' recommendations in $98 \%$ of cases with statistically significant improvement from phase 1 . Furthermore, a significant improvement in prescribing anti-emetics in accordance to NCCN guidelines was observed with an increase from $4 \%$ in phase 1 to $44 \%$ in phase $2(\mathrm{p}<0.001)$. An improvement in anti-emetics administration time documentation according to prechemo and post-chemo orders was also detected with an increase from $34 \%$ in phase 1 to $82 \%$ in phase $2(\mathrm{p}<0.001)$ (Tables 1, 2 and 3).
Upon completing trend tests, all p-values were found to be statistically significant $(\mathrm{p}<0.001)$ showing a trend towards continuous improvement with time. Two exceptions to the trend of continuous improvement were noted with the antiemetics' confirmation with the NCCN guidelines and the steroids prescribing.

Overall, when patient, protocol and chemotherapy parameters (19 parameters listed in Tables $1 \& 2$ and excluding congruence with NCCN guidelines) were taken into consideration, COT implementation showed an average improvement in order completion from $49.5 \%$ (handwritten orders) to $77.6 \%$ (phase 1-COT) to $87.6 \%$ (phase 2-COT) reflecting an increase of $38.1 \%$ in order completeness between baseline and phase 2 .

\section{Step 5-hospitals survey}

All 30 hospitals completed the survey (100\% response rate) after 2 electronic reminders and a phone call followup. The selected hospitals were located in the 6 different Lebanese districts with a bed capacity ranging between 52 and 430 beds and a combination of private and public hospitals. Five of the 30 hospitals were university medical centers. Of the 25 private and 5 public hospitals, 19 hospitals (63\%) still had their chemotherapy orders handwritten by oncologists, while 10 hospitals (33\%) had 
Table 4. Hospitals Survey

\begin{tabular}{|c|c|c|c|c|c|}
\hline Hospital & Bed- Capacity & $\begin{array}{c}\text { Public or Private } \\
\text { Sector }\end{array}$ & $\begin{array}{c}\text { Hand-Written (0) or Pre- } \\
\text { Printed Chemotherapy } \\
\text { Order Templates }\end{array}$ & $\begin{array}{c}\text { Year Pre-Printed } \\
\text { Chemotherapy Order } \\
\text { Templates (COT) Started }\end{array}$ & Future Plans \\
\hline 1 & 430 & Public & 0 & - & None \\
\hline 2 & 420 & Private & 1 & 2010 & CPOE in 5 years \\
\hline 3 & 400 & Private & 1 & 2010 & $\mathrm{CPOE}$ in 2 years \\
\hline 4 & 325 & Private & 1 & 2013 & None \\
\hline 5 & 305 & Private & 0 & - & None \\
\hline 6 & 250 & Private & 1 & 2005 & None \\
\hline 7 & 220 & Public & 0 & - & None \\
\hline 8 & 205 & Public & 1 & 2000 & None \\
\hline 9 & 203 & Private & 0 & - & CPOE \\
\hline 10 & 200 & Private & 0 & - & None \\
\hline 11 & 175 & Private & 1 & 2005 & None \\
\hline 12 & 175 & Private & 0 & - & None \\
\hline 13 & 160 & Private & 0 & - & CPOE \\
\hline 14 & 150 & Private & 1 & 2012 & CPOE \\
\hline 15 & 150 & Private & 1 & 1980's & CPOE \\
\hline 16 & 150 & Private & 0 & - & None \\
\hline 17 & 140 & Private & 0 & - & None \\
\hline 18 & 130 & Private & 0 & - & Pre-printed COT \\
\hline 19 & 130 & Private & 0 & - & Pre-printed COT \\
\hline 20 & 125 & Private & 1 & 2011 & None \\
\hline 21 & 122 & Private & 0 & - & None \\
\hline 22 & 106 & Private & 1 & 2006 & CPOE \\
\hline 23 & 80 & Private & 0 & - & None \\
\hline 24 & 80 & Private & 0 & - & None \\
\hline 25 & 80 & Private & 0 & - & None \\
\hline 26 & 75 & Private & 0 & - & None \\
\hline 27 & 71 & Private & 0 & - & None \\
\hline 28 & 70 & Public & 0 & - & None \\
\hline 29 & 65 & Private & 0 & - & Pre-printed COT \\
\hline \multirow[t]{2}{*}{30} & 52 & Public & 0 & - & None \\
\hline & & $\begin{array}{c}5 \text { Public and } 25 \\
\text { Private Hospitals }\end{array}$ & 19 Handwritten orders & & \\
\hline
\end{tabular}

COPE: Computerized Physician Order Entry, -: pre-printed COT not started at corresponding hospital

implemented pre-printed COT. One of the public hospitals did not have an oncology service. One of the hospitals developed pre-printed COT back in the 1980s, while the remaining nine hospitals had initiated COT use in the last decade. Approximately, 33\% of the surveyed hospitals planned to implement CPOE or pre-printed COT in the near future (Table 4).

\section{Lessons Learned}

This study showed that standardized chemotherapy orders significantly improve the quality of cancer care delivery by ensuring completeness of orders, reducing variations and applying best practices following established guidelines. Missed information documented in the baseline retrospective review of 100 chemotherapy orders (step 1) included allergies, protocol name and reference, total number of cycles, start date of chemotherapy, generic chemotherapy drug name, dose in $\mathrm{mg} / \mathrm{m}^{2}$, administration route, method and frequency, duration of infusion, sequence of administration of agents, pre-medications, and hydration. Such omissions in the chemotherapy orders can significantly affect the cancer care delivery and increase the potential for medication errors (Dumasia et al., 2006Hartel et al., 2011; Fyhr and Akselsson, 2012). Our study results are similar to those of Mathaiyan et al. assessing prescription errors in 1500 handwritten chemotherapy prescription orders and reporting 4253 prescription errors $(283.5 \%)$ the majority of which were due to omissions in the chemotherapy orders $(47.1 \%)$. These omissions included patient's name, age, diagnosis, pre-medications and chemotherapy related variables (dosage form, name, units of dose, diluent, route and time of administration). Mathaiyan et al. estimated that $11.7 \%$ of these errors were potentially harmful and likely to cause serious consequences to the patients (Mathaiyan et al., 2015). In our study, the postimplementation assessment phases $1 \& 2$ demonstrated that these omission errors in chemotherapy orders can be rectified with the implementation of COT through 
the pre-printed information and by ensuring physicians' thoroughness in order completion.

Our study findings are slightly more positive than those reported by Dumasia et al. whereby pre-printed chemotherapy orders improved prescription completeness from $45 \%$ with handwritten orders to $81 \%$ with standardized pre-printed orders reflecting an improvement of 36\% (Dumasia et al., 2006). Similarly, Meisenberg et al. documented the number and type of errors associated with chemotherapy order composition related to three sequential methods of ordering: handwritten orders, preprinted orders, and CPOE. From 2008 to 2012, samples of completed chemotherapy orders were reviewed by a pharmacist for the number and type of errors as part of routine performance improvement monitoring. Error frequencies for each of the three distinct methods of chemotherapy orders were compared and the rate of problematic order sets (those requiring significant rework for clarification) was reduced from $30.6 \%$ with handwritten orders to $12.6 \%$ with pre-printed orders (preprinted vs handwritten, $\mathrm{p}<0.001$ ) then further to $2.2 \%$ with CPOE (pre-printed vs CPOE, $\mathrm{p}<0.001$ ). The incidence of errors capable of causing harm was also reduced from $4.2 \%$ with handwritten orders to $1.5 \%$ with pre-printed orders (pre-printed vs handwritten, $\mathrm{p}<0.001$ ) to $0.1 \%$ with CPOE (CPOE vs pre-printed, $\mathrm{p}<0.001$ ) (Meisenberg et al., 2014).

The dual review phase completed in this study is in compliance with the ISMP guidelines that recommend at least biannual review of the orders to ensure that no more than two years have lapsed since their approval (ISMP, 2014). Prior to order implementation, educational grand presentations and meetings of concerned hospital committees were crucial in setting the stage for change in practice and cultivating a team approach as recommended by Kotter in his 8 - step process for leading change (Kotter International, 2015). Findings from phase 1 were a motive for further hospital staff engagement in the process and in creating a sense of urgency for change. Physicians and nurses' engagement in educational sessions and meetings for feedback collection and barriers identifications were crucial in gaining their acceptance of this change and embracing the new practice. According to their input, orders were modified for clarity, enhancement, and better compliance. As a result, the improvements seen between phases 1 and 2 are noteworthy and included completeness of order parameters requiring prescribers' compliance with the COT (breaching of the protocols as per NCCN guidelines' recommendations decreased from $37 \%$ in phase 1 to $2 \%$ in phase 2 ) and thoroughness (completing all patient specific information such as allergies, height and weight and protocol specific information such as cycle number and total number of cycles). Similar to our findings post-implementation of COT, a study that reviewed prescribing errors in oncology showed that missed information $(19.86 \%)$, errors in the calculation of chemotherapy dose $(19.14 \%)$ and protocol breach $(13.71 \%)$ were the most frequent errors, followed by hydration $(6.86 \%)$ and omission $(4.14 \%)$ in pre-printed COT (Hamza et al., 2013).

Despite order standardization, there were still opportunities for improvement. For example, prescribers were confused regarding the start date of chemotherapy as this information was required in two different locations on the pre-printed orders which triggered modification of orders to address this confusion $(71 \%$ of the orders in phase 1 had the start date documented compared to $100 \%$ of the orders in phase 2 once modifications were implemented). Other recommendations to improve the orders included adding monitoring parameters, hold parameters, frequency of re-weighing the patient for weight documentation, and cumulative lifetime dose when applicable.

It is worth noting that the use of anti-emesis agents use was poorly compliant with the NCCN recommendations all through the multiple phases of this study. The evaluated orders in phase 1 were in line with the guidelines in $4 \%$ of the cases only. A statistically significant improvement was seen from phase 1 to phase 2 where $44 \%$ of the chemotherapy orders analyzed were in congruence with the NCCN guidelines. The major barrier to compliance with the guidelines was the lack of supportive care resources for anti-emesis in Lebanon. In fact, the neurokinin-1 antagonist aprepitant is not available in Lebanon and fosaprepitant was first imported to the country in June 2012. Moreover, the 5-HT3 antagonist, palonosetron, which was preferred among this class of agents, is not available in Lebanon. In addition, the newly approved combination of netupitant/palonosetron is also not yet available. Thus, preventing the risk of emesis with high-emetic potential regimens was impossible to achieve and oncologists were faced with limited options to support patients through this complication. This lack in important supportive care medications might explain why oncologists used various combinations of steroids, 5HT3 antagonists and other antiemetic agents leading to breach of protocols. It is also worth noting that many of the supportive care agents are not covered by the National Social Security Fund (NSSF) leaving the patients with added financial restrains. Similarly, Zeitoun et al. documented the inadequate adherence to NCCN guidelines for anti-emetics use in 15 different Lebanese hospitals with approximately $40 \%$ of enrolled patients receiving inappropriate anti-emetic regimens (Zeitoun and Nassif, 2013).

Worldwide, many hospitals still base the drug prescription and administration process on handwritten medical chart entries (Hartel et al., 2011). Based on the hospitals survey that was completed in step 5, many hospitals in Lebanon also rely on handwritten orders. Accordingly, this study could serve as an incentive to the many hospitals that are still using handwritten orders to move to pre-printed COT or CPOE if the resources are available. It also could be of added value to the hospitals that are in the phases of implementing COT or planning to do so as part of CPOE by providing them with the steps for implementation and barriers to overcome. In fact, the study findings will be presented to the Ministry of Public Health in Lebanon to assist in moving all hospitals from handwritten to pre-printed COT. The study did not assess the consequences of these omission errors in chemotherapy orders on the patients or the ensuing cost 
burden.

Safety experts recommend the use of pre-printed orders and technology to decrease errors such as CPOE, bar-coding and automation. CPOE is associated with low error rates, but does not eliminate all errors. Technology, itself, can introduce novel types of errors not seen with traditional handwritten or pre-printed orders (Hartel et al., 2011; Hamza et al., 2013). Accordingly, vigilance, even with CPOE, is still required to avoid patient harm. Knowing that many Lebanese hospitals have limited financial and technical resources and are unlikely to afford CPOE implementation, pre-printed orders could be a step forward in securing chemotherapy orders completeness and providing quality patient care through standardization.

\section{Conclusion}

Chemotherapy orders completeness was improved by pre-printed chemotherapy order templates. Standardizing the chemotherapy orders lead to quality improvement in prescribers' inclusion of necessary patient, chemotherapy and supportive care order variables and to a decrease in the risk of medication errors. By engaging the health care providers, chemotherapy order templates were successfully implemented as a standard of care for the cancer patient population.

\section{Acknowledgements}

The authors would like to acknowledge Hanane Wakim, Pharm.D. for her assistance in the study and Jeanette Nassif, Pharm.D. for her review of the manuscript.

\section{References}

ASHP (2002). guidelines on preventing medication errors with antineoplastic agents. Am J Health Syst Pharm, 59, 1648-68.

Birner AM, Bedell MK, Avery JT, Ernstoff MS (2006). Program to support safe administration of oral chemotherapy. J Oncol Pract, 2, 5-6.

Dreyfuss JH (2010). New joint outpatient chemotherapy administration standards. CA Cancer J Clin, 60, 66-7.

Dumasia L, Harris E, Drelichman A (2006). Quality performance improvement with the implementation of standard chemotherapy order forms. J Oncol Pract, 2, 104-7.

Fyhr A and Akselsson R (2012). Characteristics of medication errors with parenteral cytotoxic drugs. Eur J Cancer Care (Engl), 21, 606-13.

Hamza NA, Sallam SA, El-Nimr NE (2013). Rates and types of prescribing errors and related interventions in oncology. Int J Curr Microbiol App Sci, 2, 435-45.

Harris T and Northfelt DW (2005). Chemotherapy error: practical approaches to increasing patient safety. $J$ Patient Saf, 1, 215-9.

Harris N, Badr LK, Saab R, Khalidi A (2014). Caregivers' perception of drug administration safety for pediatric oncology patients. J Pediatr Oncol Nurs, 31, 95-103.

Hartel MJ, Staub LP, Roder C, Eggli S (2011). High incidence of medication documentation errors in a Swiss university hospital due to the handwritten prescription process. BMC Health Serv Res, 11, 199.

Institute for Safe Medication Practices (ISMP) ISMP Guidelines (2014). http://www.ismp.org/Tools/guidelines/default.asp.
Kloth DD (2010). Guide to the Prevention of Chemotherapy Medication Errors: Strategies To Prevent Chemotherapy Errors $2^{\text {nd }}$ edition, Clinical Oncol, .

Li EC, Hinkel JM, Gallagher L, et al (2009). NCCN chemotherapy order templates: enhancing patient safety through standardization. J Clin Oncol, 27, 17537.

Mathaiyan J, Jain T, Dubashi B, Reddy KS, Batmanabane G (2015). Prescription errors in cancer chemotherapy: Omissions supersede potentially harmful errors. J Pharmacol Pharmacother, 6, 83-7.

Meisenberg BR, Wright RR (2014). and Brady-copertino CJ: Reduction in chemotherapy order errors with computerized physician order entry. J Oncol Pract, 10, 5-9.

Northfelt DW, Allbritton DW, Parra LS, et al (2003). Medication errors in cancer therapy: review and proposal for systematic study. Proc Am Soc Clin Oncol, 22, 542.

Phillips J, Beam S, Brinker A, et al (2001). Retrospective analysis of mortalities associated with medication errors. Am J Health Syst Pharm, 58, 1835-41.

Ranchon F, Salles G, Spath HM, et al (2011). Chemotherapeutic errors in hospitalised cancer patients: attributable damage and extra costs. BMC Cancer, 11, 478.

Shaikh BS, Mowat RB, Parr DD (2004). Chemotherapy ordering process: Safety and efficiency considerations. J Clin Oncol, 22, 6110 .

Schulmeister L (2006) Preventing chemotherapy errors. Oncologist, 11, 463-8.

Serrano-Fabia A, Albert-Mari A, Almenar-Cubells D and Jiménez-Torres NV (2010). Multidisciplinary system for detecting medication errors in antineoplastic chemotherapy. J Oncol Pharm Pract, 16, 105-12.

The 8-Step Process for Leading Change (2015). Available at: http://www.kotterinternational.com/the-8-step-process-forleading-change/. Accessed August 12, 2015.

Walsh KE, Dodd KS, Seetharaman K, et al (2009). Medication errors among adults and children with cancer in the outpatient setting. J Clin Oncol, 27, 891-6.

Zeitoun AA, Nassif JG (2013). Evaluation of anti-emetic use in chemotherapy-induced nausea and vomiting in a third world country (Lebanon). J Eval Clin Pract, 19, 68-75. 